\title{
Acquisition of Animated and Pop-Up Targets
}

\author{
Guillaume Faure $^{1,2}$, Olivier Chapuis ${ }^{1,2}$, and Michel Beaudouin-Lafon ${ }^{1,2}$ \\ ${ }^{1}$ LRI -- Univ. Paris-Sud \& CNRS, Orsay, France \\ ${ }^{2}$ INRIA, Orsay, France
}

\begin{abstract}
Pop-up targets, such as the items of popup menus, and animated targets, such as the moving windows in Mac OS X Exposé, are common in current desktop environments. This paper describes an initial study of pointing on popup and animated targets. Since we are interested in expert performance, we study the situation where the user has previous knowledge of the (final) position of the target. We investigate the effect of the DELAY factor, i.e. the delay before the target pops up (for pop-up targets) or the duration of the animation (for animated targets). We find little difference between the two techniques in terms of pointing performance (time and error), however a kinematic analysis reveals differences in the nature of the pointing movement. We also find that movement time increases with DELAY, but the degradation is smaller when the target is farther away than when it is closer. Indeed, larger distances require a longer movement time therefore the target reaches its destination while the participant is still moving the pointer, providing more opportunity to correct the movement than with short distances. Finally we take into account these results to propose an extension to Fitts' Law that better predicts movement time for these tasks.
\end{abstract}

Keywords: Pop-up targets, Animated targets, Movement analysis, Fitts'.

\section{Introduction}

Pointing is a fundamental action in graphical user interfaces (GUIs) that has been the subject of much research to both understand pointing actions and improve pointing techniques. Most of this research has focused on static targets that are displayed before pointing starts and stay still during the pointing action. GUIs however have always featured pop-up targets that appear after the pointing movement starts, e.g., pop-up menus and dialog boxes. More recently, GUIs have also started to feature animated targets that move while the pointing gesture is being performed, e.g., the windows in Mac OS X Exposé. While we know that animation enhances user interaction with the system $[13,24]$ by providing a continuous feedback that increases the user's sense of direct and indirect interaction [26], its effect on pointing has not, to the best of our knowledge, been studied. In particular, beyond the empirical evidence that users take advantage of their knowledge of the behaviour of targets to anticipate their apparition or final position, we are not aware of any systematic study of this phenomenon.

This paper presents what we believe is the first controlled study designed to better understand the acquisition of animated and pop-up targets. Our main factor is DELAY, the delay before the target pops up (for pop-up targets) or the duration of the 
animation (for animated targets). We have focused on short values of DELAY between 0 and 500ms - which are typical of GUIs, and we have operationalized the common situation where users can anticipate the final position of the target by ensuring that they have prior knowledge of its location.

The experiment compares pointing performance (in time and error) for static, animated and pop-up targets under various values of DELAY. We found a strong effect of DELAY: movement times increase with the delay, but the degradation is smaller for longer distances to target than for shorter ones. We also found little performance difference between pop-up and animated targets, but an interesting qualitative difference when looking at the kinematic profiles. In particular, this analysis shows evidence of how users anticipate the final position of the target.

Finally we show that Fitts' Law does not accurately predict movement times for pop-up and animated targets. Using the insights gained by the analysis of the experimental results, we propose an extension to Fitts' Law that takes into account the unique characteristics of these tasks to better predict movement time.

The rest of the paper is organized as follows. After some background and related work, the next section provides a set of examples and motivates the present study. The subsequent sections describe the experiment, present the performance results (movement time and errors), analyse the kinematic aspects of typical movements (velocity vs. distance and number of sub-movements) and propose an extension to Fitts' Law. The paper then concludes with a discussion and directions for future work.

\section{Background and Related Work}

The acquisition of an on-screen target by means of a pointing device (pointing for short) is one of the basic tasks in Graphical User Interfaces and one that has been extensively studied in Human-Computer Interaction (HCI). Previous research in this area includes novel techniques to facilitate target acquisition, comparison of input devices performance, and models to predict movement time and better understand target acquisition tasks of various types. The major theoretical tool for studying pointing is Fitts' Law [11], which predicts the movement time to acquire a target of width W at a distance D. The most widely used form of Fitts' Law in HCI [19] is:

$$
M T=a+b \log _{2}\left(\frac{D}{W}+1\right)
$$

where $a$ and $b$ are empirically determined constants and where $\log _{2}(D / W+1)$ is called the index of difficulty (ID) of the task. While a number of variations of this law have been proposed (see [23] for a review), the Psychology literature has not reached a consensus yet on an explanation of this Law.

The most popular explanation of Fitts' Law in the HCI community is Meyer et al.'s sub-movements model [20]: first, a fast sub-movement towards the target (the ballistic phase of the movement) is performed, then if this movement does not hit the target, another (probably smaller) sub-movement towards the target is performed, and this process continues until the target is reached. An important characteristic of the sub-movements model that may explain the logarithmic form of Fitts' Law is that the distance vs. velocity graph shows a bell shape with a clear velocity peak. 
The originality of our study is that the target is not always visible on the screen during the acquisition movement. Most previous work in HCI has investigated the acquisition of an initially invisible target using navigation tasks, e.g., scrolling and pan-and-zoom, rather than a pure pointing task. A notable exception is Cao et al. [7] where the targets are initially invisible and are revealed by moving a display window attached to the cursor (coupled cursor). The pointing task in that case involves two phases: first, reveal the target, then acquire it. In our study the targets are revealed automatically (without user action) and users know in advance where the targets are so they can anticipate their final position. We therefore expect a more integrated motion than in the above study, with dynamic adaptation to the target behaviour.

Some studies have shown that the shortest delay needed for sensory information to affect hand movement is approximately $100 \mathrm{~ms}$ [17]. For example Flash and Henis [12] have observed movement adaption between 100 and $200 \mathrm{~ms}$ after an experimentally controlled modification of the cursor trajectory. Closer to HCI, Zhai et al. [28] have shown that users can take advantage of unpredictable target expansion, suggesting a dynamic adaptation of the user's pointing movement.

Menus are the main example of pop-up targets and have been widely studied in HCI. With linear menus, it is assumed that the menu pops up immediately and studies have focused on factors such as the number of items and the depth of the hierarchy of menus rather that the pop-up delay (see, e.g., [9]). With marking menus [18] and their variants, there is a distinction between novice mode, where the user waits for the menu to pop up, and expert mode, where the menu does not appear at all. Here, the absence of motion during the delay is used to activate novice mode (see also [14]).

Regarding animated targets, previous studies in Psychology, e.g., [15], and in HCI [21] have addressed the acquisition of moving targets. These studies however have considered capturing tasks, i.e. acquiring the target while it is moving whereas in our case the target can be acquired only when it has reached its destination. Other examples that involve moving targets include facilitation techniques that bring the targets (with a possible animation) close to the cursor after the movement has started, e.g., Drag-and-pop [4] and Vacuum [5]. These techniques however make it difficult for the user to anticipate the final position of the target.

Memorization of target positions is an important point of our experimental design and has been the subject of previous work, e.g., Hornof and Kieras [16]. However, we only consider the memorization of a single target in short term memory. This phenomenon has been studied in the domain of Human Vision (see, e.g., [2]) where it has been shown that humans can recover spatial positions via saccades (rapid eye movements) from a few memorized gaze positions.

Surprisingly, we have not found in the literature any previous study of pointing on memorized "invisible" targets. The reverse paradigm however, pointing with an invisible cursor, has been widely studied (see, e.g., [6]) to better understand whether arm movements follow a position model or an amplitude model. It is interesting to note that our study seems to support the amplitude control model.

\section{Motivation and Examples}

In the rest of this article we call Anim the condition where the subject is pointing on an animated target and Popup the condition where the subject is pointing on a pop-up target. When the duration of the animation is null (DELAY $=0$ ), the Anim condition 
is equivalent to the Рорир condition: the target appears immediately when movement starts. This is different however from the acquisition of a persistent target, when the target is visible at its destination before the movement starts. This latter condition is the one usually considered in pointing experiments and will act as a control. We call it the Static condition.

One motivation for the design of the experiment was the following scenario. The user wants to drag-and-drop a hidden icon from the desk ${ }^{1}$ to an area of the working window, e.g., to attach a file to an email. The user typically must trigger a command to make the desk visible, grab the icon and then make the original working window visible again to drop the icon at the desired destination. With Mac OS X, the user may use Exposé to make the desk visible: all windows are moved outside the screen with an animation and then animated back to their original position when the user starts the drag operation. This corresponds to the Anim condition because the target of the drop operation is an animated window. With Microsoft Windows and in most modern X Window environments the user may use the "show desktop" feature to immediately remove the windows and make the desk visible, start dragging and then show the windows again. This corresponds to the Pорир condition with, ideally, a very short delay. A variant of this technique is desk pop [, 2007.

10]: the desk is moved to the foreground with a semi-transparent background, providing access to the icons while keeping the windows accessible. This case corresponds to the Static condition.

Dialog boxes are another examples of animated and pop-up targets with a potential a priori knowledge of the position of the targets by the user. Such windows often pop up at the same position and contain only a few buttons, e.g., "Ok" and "Cancel". Users often know the action to perform before the dialog box pops up, and we have observed [8] that they tend to anticipate the display of the dialog box by moving their mouse pointer toward the target button before it appears. A typical example is the "how to download" dialog box of the Firefox browser, which shows up when clicking a link to non-HTML content. Due to network delays, the box pops up with an unpredictable lag and yet users anticipate its appearance. Mac OS X features an example of animated target: some dialog boxes "slide" out of their parent window's titlebar and here too, users anticipate the location of the button they want to click.

Finally, an interesting category of pop-up targets is given by web navigation. Users often revisit the same web pages [22] and end up knowing their layout. They also often follow the same navigation paths [25]. For example, a user loads a page, clicks on the login button of a pre-filled form that loads a new page, and finally clicks on a link to navigate to the desired page. The delays involved in displaying the various pages and the targets they contain depend not only on the speed of the network connection but also on the browser's rendering algorithm and the structure of the page. Understanding the effect of delays on pointing may thus have some implications on browser and web page design and, more generally, GUIs.

\section{Experiment}

We conducted an experiment to study pop-up and animated targets by reducing the problem to a one-dimensional Fitts-like pointing task. Fitts' Law is inherently a 1D

\footnotetext{
${ }^{1}$ The background area of the screen containing icons.
} 
model and considering 2D pointing would have involved additional factors [1] that we did not want to include in this first study.

\subsection{Apparatus}

The experiment was conducted on a $2.33 \mathrm{Ghz}$ Core2-Duo Macbook Pro with an ATI Radeon X1600 graphics card connected to a 24 inch, 1920x1200 pixels LCD monitor. The experiment was implemented in Java with SwingStates [3]. We used a 1000 dpi LogiTECH RX 250 optical mouse with the default Mac OS X acceleration. The animations were linear translations computed at a rate of 60 steps by second.

\subsection{Participants}

12 unpaid adult volunteers (10 male and 2 female) participated in the experiment. All were right-handed and aged from 23 to 35 years old (average 27.6, median 26). All were experienced computer users familiar with mouse pointing.

\subsection{Task and Procedure}

A trial is a 1-D target acquisition task decomposed as follows. First, a green target is displayed on the screen (Figure 1.a) and the participant clicks on it when ready. The green target then disappears and the main target (in grey) appears for one second (Figure 1.b). This is the memorization phase where the participant is informed of the final position of the main target. After the main target disappears, a red target is displayed (Figure 1.c). In the Static condition the main target stays on screen while in the other two conditions the main target disappears. In all three conditions, the participant then has to click on the red target, which starts the recording of the movement time. In the Static condition, the main target remains static for the rest of the trial. In the Anim condition, the main target is smoothly animated to its final position in DELAY $m s$. In the Popup condition, the main target appears after DELAY $m s$. The trial ends when the participant successfully acquires the main target (Figure 1.d) after is has appeared at its final position.

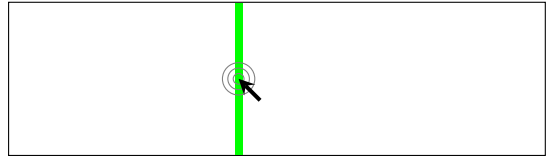

(a)

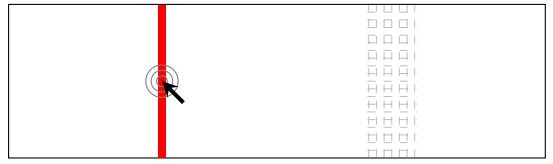

(c)

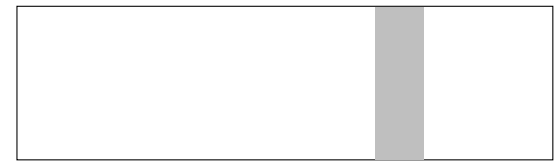

(b)

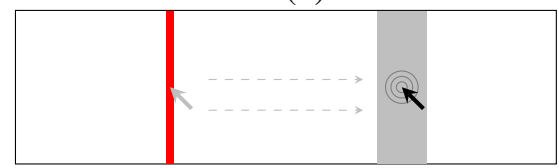

(d)

Fig. 1. (a) Start of trial: click green target; (b) memorization phase: show main target for one second; (c) target on screen (Static) or hidden (other conditions); click red target to start pointing; (d) end of trial: click main target (after animation or pop up according to condition) 
The participants' movement time is recorded from the time the mouse button is released on the red target until the successful button press on the main target. If the participant clicks outside the target or before it reaches its final position, the trial is considered an error but continues until a successful click. Participants are instructed to perform the task as fast and as accurately as possible. To prevent participants from using the cursor to memorize the position of the target the mouse pointer is hidden throughout the memorization phase. Finally, in the Anim condition, the main target moves from the closest border of the screen to its final position in the opposite direction to the acquisition movement.

\subsection{Design}

The experiment is a partial $2 \times 5 \times 3 \times 3$ within-participant design with the following factors:

- 2 "techniques"conditions (TECH): Anim and Popup;

- 5 "delays" conditions (DELAY): Static and 0, 200, 350 and 500 milliseconds $(\mathrm{ms})$ representing the duration of the animation or the delay for the pop-up;

- 3 widths (W) for the main target: 16, 32 and 64 pixels;

- 3 acquisition distances (D) to the main target: 256, 512 and 768 pixels.

The design is only partial (not fully factorial) because crossing TECH and DELAY leads to only $2+2 \times 3=8$ conditions since the DELAY conditions Static and 0 are the same for both TECH conditions: an animation of $0 \mathrm{~ms}$ makes the target pop up immediately. Static is not really a "delay" condition but it is a convenient way to consider it as both a control and an extreme condition (note that this transforms DELAY into a nominal factor, but we will later transform it into a continuous one).

We use $350 \mathrm{~ms}$ as the median animation time because this duration is commonly used in graphical user interface animations, e.g., Mac OS X Exposé. We ran pilot studies in order to determine the range of reasonable animation and pop-up times, resulting in 200ms for the lower bound and 500ms for the upper bound.

We divide a run of the experiment into two parts. The first is made of two blocks, one with DELAY=Static and the other with DELAY=0. The second part of the experiment corresponds to the crossing of TECH (Anim and Popup) with the DELAY conditions $>0$, i.e., $2 \times 3=6$ blocks. We block by the TECH condition and use a $3 \times 3$ latin square to cross the 2 possible orders of TECH with the 3 non-zero DELAY conditions. This gives 6 counter-balanced orders that we cross with the first two blocks. We divide the 12 participants into two groups of 6 . Both groups use these 6 orders, but the first group starts with DELAY=Static while the second group starts with DE$\mathrm{LAY}=0$.

Each of the 8 blocks described above has 7 replications of the $3 \times 3=9$ combinations of $\mathrm{D}$ by $\mathrm{W}$, presented in a random order. The first replication is considered a warm up. A pause is offered to participants at the beginning of each replication.

To summarize, the total number of logged trials in the experiment is 8 blocks $\times 9$ width-distance combinations $\times 6$ replications $\times 12$ participants $=5184$ trials. $\mathrm{We}$ logged 72 trials for each full condition, 6 for each participant. The experiment lasted from 42 to 54 minutes (average 48, median 47). 


\section{Results}

In this analysis, movement time MT is measured until a successful button press in the target (as opposed to the first button press). This has the advantage of accounting for penalties caused by errors. We duplicate data for Static and DELAY $=0$ in order to simulate these conditions for both Popup and Anim. This allows us to perform a standard full-factorial repeated measures analysis of variance MT $\sim \mathrm{TECH} \times \mathrm{DELAY} \times \mathrm{D}$ $\times \mathrm{W} \times$ Random(Participant). Outliers ${ }^{2}$, which represent $0.35 \%$ of the trials, are removed from all analyses ${ }^{3}$.

Table 1. ANOVA for MT TECH $\times$ DELAY $\times \mathrm{D} \times \mathrm{W} \times$ Random(Participant)

\begin{tabular}{|c|c|c|c|c|}
\hline Factors & DF & DFDen & F & P \\
\hline TECH & 1 & 22 & 4.66 & 0.0539 \\
DELAY & 4 & 44 & 97.74 & $<0.0001$ \\
D & 2 & 22 & 68.63 & $<0.0001$ \\
W & 2 & 22 & 481.94 & $<0.0001$ \\
\hline TECH $\times$ DELAY & 4 & 44 & 1.43 & 0.2396 \\
TECH $\times$ D & 2 & 22 & 0.19 & 0.8303 \\
TECH $\times$ W & 2 & 22 & 1.05 & 0.3653 \\
DELAY $\times$ D & 8 & 88 & 8.33 & $<0.0001$ \\
DELAY $\times$ W & 8 & 88 & 1.90 & 0.0686 \\
D $\times$ W & 4 & 44 & 2.67 & 0.0444 \\
\hline TECH $\times$ DELAY $\times$ D & 8 & 88 & 0.19 & 0.9920 \\
TECH $\times$ DELAY $\times$ W & 8 & 88 & 0.35 & 0.9436 \\
TECH $\times$ D $\times$ W & 4 & 44 & 0.51 & 0.7274 \\
DELAY $\times$ D $\times$ W & 16 & 176 & 1.11 & 0.3469 \\
\hline TECH $\times$ DELAY $\times$ D $\times$ W & 16 & 176 & 1.05 & 0.4071 \\
\hline
\end{tabular}

Table 1 shows the results of the repeated analysis of variance for movement time. As expected $\mathrm{D}$ and $\mathrm{W}$ have a (strong) significant effect on movement time: the harder the task, the longer it takes to complete (Figure 2). TECH fails to reach significance for movement time and we find no significant interaction with the other factors. The difference in mean between Anim and Popup is only $17 \mathrm{~ms}$ in favor of Popup. This difference (see Figure 3 ) is less than $2 \%$ of the mean movement time ${ }^{4}$.

We observe a (strong) significant effect of DELAY on movement time (Figure 3 ). A Tukey post-hoc test $(\alpha=0.05)$ shows no significant difference in mean between Static and DELAY $=0$ nor between DELAY $=0$ and DELAY $=200$. However, it shows a significant difference in mean between Static and DELAY $=200(74 \mathrm{~ms}$ in favor of Static, a speed-up of 7.7\%). It also shows that DELAY $=200$ is significantly faster than DELAY $=350(120 \mathrm{~ms}, 11.1 \%$ speed-up $)$ and that DELAY $=350$ is significantly faster than DELAY $=500$ (126 $\mathrm{ms}, 10.4 \%$ speed-up).

We also observe a significant interaction effect between DELAY and D (Figure 2.a), suggesting that the effect of distance on movement time is less strong as

\footnotetext{
${ }^{2}$ Defined as a movement time 2 standard deviations a away from the mean movement time (for each PARTICIPANT, TECH, DELAY, D and W).

${ }^{3}$ Including the outliers yields results that are very similar to those described here.

${ }^{4}$ Note that the data duplication for Static and DELAY $=0$ does not influence these statistical results since they cancel each other out.
} 


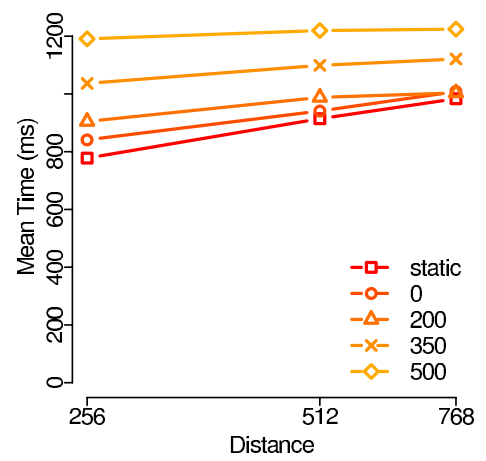

(a)

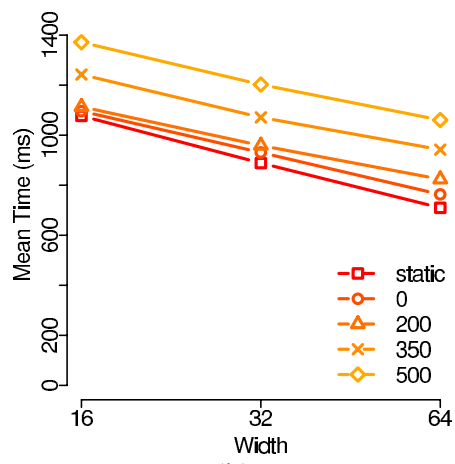

(b)

Fig. 2. (a) Movement time as a function of distance for each DELAY condition. (b) Movement time as a function of width for each DELAY condition.

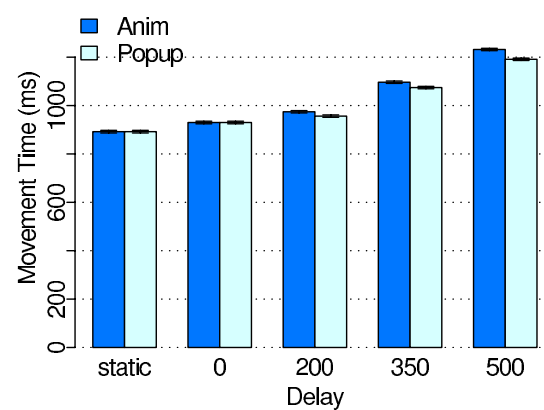

(a)

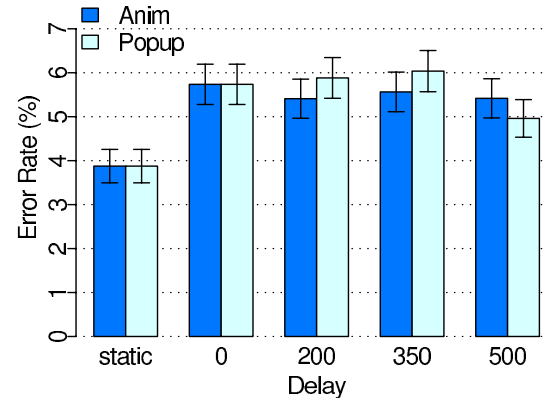

(b)

Fig. 3. Movement time (a) and Error rate (b) for each DELAY and TECH

DELAY increases from Static to DELAY $=0$ to DELAY $=500$. Indeed, a post-hoc Tukey test $(\alpha=0.05)$ shows that there is a significant difference (in mean) between each distance for Static and DELAY $=0$, a significant difference between distances 256 and 512 but not between distances 512 and 768 for DELAY $=200$, a significant difference between distances 256 and 768 but not between distances 256 and 512 and distances 512 and 768 for DELAY $=350$, and finally no significant difference at all for DELAY $=500$.

Figure 2.a also suggests that the difference in movement time between Static, DELAY $=0$ and DELAY $=200$ decreases as distance increases. Indeed, a post-hoc Tukey test shows a significant difference in mean between Static and DELAY $=200$ for $\mathrm{D}=256$ but no such difference for $\mathrm{D}=768$.

The above phenomenon can be explained by the fact that participants start their acquisition movement as soon as possible, i.e., before the end of the animation or before the target pops up, and that this ballistic movement is more precise when the target pops up or stops its animation earlier. For example, with DELAY $=500$ and D $=256$, participants can typically move the pointer close to the target before it pops up (or finishes its animation), but then have to wait before performing the final adjustment. On the other hand, with DELAY $=200$ and D $=768$, the target pops up (or finishes its 
animation) before the end of the ballistic movement and participants can adjust their movement as they go.

Regarding errors, we measured an error rate of $5.25 \%$, a typical value for a pointing experiment. Figure 3.b shows the error rate as a function of DELAY for each TECH. Logistic two by two Pearson tests ${ }^{5}$ show a significant difference $\left(\chi^{2}=4.880\right.$, $p=0.0272$ for DELAY $=0$ ) between Static (an error rate of $3.87 \%$ ) and the other DELAY conditions (error rates between $5 \%$ and $6 \%$.) There is no significant difference for errors among the remaining DELAY conditions. Other Pearson tests did not reveal any significant effect on errors for the other factors TECH, D and W.

\section{Kinematic Analysis}

A more detailed analysis of the pointing movements shows that participants effectively start their movement before the target pops up (when TECH = Popup and DELAY $>0$ ) or before the target ends its animation (when TECH $=$ Anim and DELAY > 0 ). Figure 4.a shows the time taken by the participants between the mouse button release (when they click on the red starting target) and the first mouse movement. These are clearly shorter than DELAY when DELAY > 0. Moreover, an ANOVA shows no significant difference between the DELAY conditions for TECH = Popup, nor between the non-zero DELAY conditions for TECH = Anim. However, as shown in Figure 4.a, the first mouse move times for TECH $=$ Anim and DELAY $>0$ are significantly longer than those for the other conditions $(\sim 40 \mathrm{~ms})$.

An analysis of the kinematic record of the movements shows that Popup and Anim have slightly different profiles (see Figure 5 for an example, similar shapes arise with the other distances). The distance/speed curves for Popup are similar to those for the Static and DELAY $=0$ conditions: they all are bell-shaped with a velocity peak at about $50 \%$ of the distance. The curves for Anim however are qualitatively different: they are right-skewed with a lower acceleration and a velocity peak at about $60 \%$ of the distance to target (these differences are statistically significant). Moreover, in the

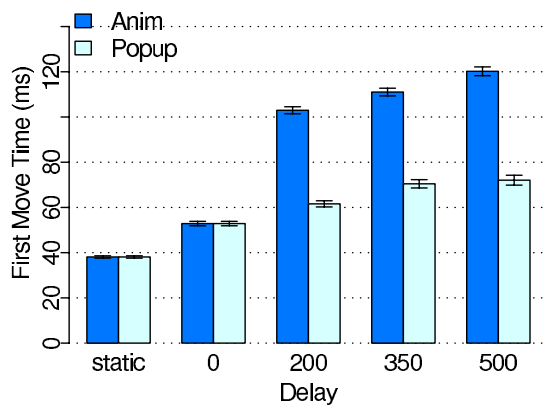

(a)

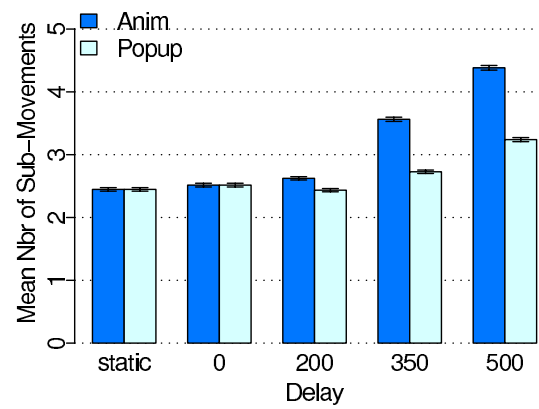

(b)

Fig. 4. (a) Time before first mouse move as a function of DELAY and TECH. (b) Mean number of sub-movements as a function of DELAY and TECH.

\footnotetext{
${ }^{5}$ Tests adapted to "discrete" measures.
} 


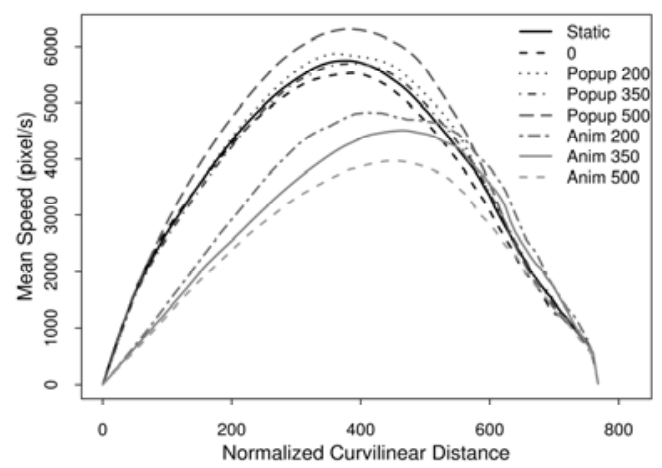

Fig. 5. Mean speed as a function of the (normalized curvilinear) distance for $D=768$ for each DELAY $\times$ TECH condition (grey curves with a lower velocity peak are the Anim curves)

Anim condition we observe slower movements and in particular a lower velocity peak as DELAY increases. In the Popup condition we do not observe any such pattern.

A possible explanation of these phenomena is that in the Anim condition the participants try to follow the moving target or are distracted by it, leading to a slower movement. Conversely, in the Popup condition, participants move directly to the memorized position of the target, leading to a movement close to the classical target acquisition profile (note that in Figure 5.a the movement is even faster for DELAY = 500 , but this is not the case for the other distances).

To confirm the above interpretation and to better understand the end of the movement we computed the number of sub-movements for all non-error trials. To do so, we count how many times the mouse does not move during at least $50 \mathrm{~ms}$ (to account for a null velocity) after the velocity peak and before the final mouse press. We add one to this number to account for the final movement, and interpret the result as a good estimate of the number of sub-movements. Figure 4.b shows the mean value of this number as a function of TECH and DELAY. An analysis of variance shows a significant effect of TECH and DELAY and an interaction between these two factors $^{6}$. There are more sub-movements for Anim than for Popup and the difference (about one sub-movement) is significant for DELAY $>200$.

In summary, we found that while Popup and Anim have similar performance, the pointing motion for Popup is closer to static pointing than to Anim. While Popup seems to follow a simple amplitude control model (jump to the anticipated position of the target and then adjust), Anim features a more complex movement.

\section{Extending Fitts' Law}

In order to better understand the effect of DELAY we used Fitts' Law to analyze movement time. Table 2 shows the Fitts' Law parameters and the adjusted $r^{2}$ for all data by DELAY. We averaged movement times across participants, repetitions and

\footnotetext{
${ }^{6} \mathrm{D}$ and $\mathrm{W}$ also have significant effects - more sub-movements for smaller $\mathrm{W}$ and more submovements for $\mathrm{D}=512$ and 768 than for 256 - but there is no significant interaction effect with TECH and DELAY.
} 
TECH since we have shown that this factor did not have a significant effect on MT. In other words, we take the mean of all trials for each DELAY, D and W conditions. As we expected, the fit for the complete dataset is not good because of a strong effect of DELAY (Figure 6.a). The fit for each DELAY condition is good for the Static condition (traditional Fitts' pointing) but it degrades as DELAY increases because of the unusual effect of distance on movement time. The fit for each DELAY condition can be improved by using Welford's model [27] $M T=a+b \cdot \log _{2}(D)+b \cdot \log _{2}(W)\left(r^{2}=\right.$ $0.95)$, but this model fails to properly fit the complete dataset $\left(r^{2}=0.545\right)$.

In order to compute regression models that include DELAY, we create a continuous factor TV as follows: We map Static to $0 \mathrm{~ms}$; We map DELAY $=0$ to $100 \mathrm{~ms}$ since this is the delay needed for sensory information to affect the physical movement [17]; Finally we map the other values of DELAY to themselves.

We can now consider the simple model $M T=a+b . I D+c . T V$, which dramatically improves the fit: $M T=307+135 . I D+0.64 . T V$, adjusted $r^{2}=0.8921$. Using Welford's model instead: $M T=a+b \cdot \log _{2}(D)+c \cdot \log _{2}(W)+d . T V$ improves the fit: $M T=977+74 . \log _{2}(D)-158 \cdot \log _{2}(W)+0.64 . T V$, adjusted $r^{2}=0.9478$. This can be further improved to $r^{2}=0.9665$ by adding yet another term, $\log _{2}(D) * T V$, to account for the D by DELAY interaction observed in the previous section.

However good the fit, none of these models are particularly intuitive. They simply assume a linear effect of DELAY and an unusual interaction of DELAY with Distance. The problem with this approach however is that the resulting model has four free variables $(a, b, c, d)$ while we have only three main factors (D, W, DELAY). If we are to be consistent with Fitts' Law, we should have at most three variables.

Table 2. Fitts' Law parameters $M T=a+b . I D$

\begin{tabular}{|c||c|c|c|c|}
\hline DELAY & $\mathrm{a}$ & $\mathrm{b}$ & adj. $r^{2}$ & \# pts. \\
\hline \hline ALL & 454 & 135 & 0.4998 & 45 \\
\hline \hline Static & 180 & 174 & 0.9656 & 9 \\
0 & 299 & 153 & 0.9074 & 9 \\
200 & 462 & 120 & 0.8973 & 9 \\
350 & 599 & 116 & 0.7974 & 9 \\
500 & 731 & 115 & 0.6654 & 9 \\
\hline
\end{tabular}

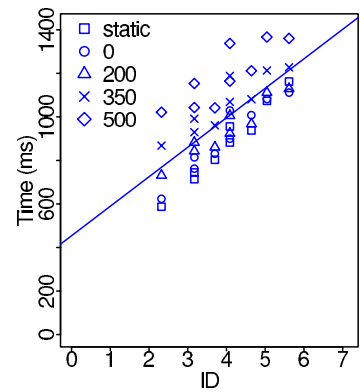

(a)

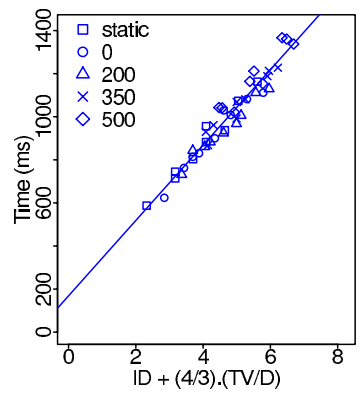

(b)

Fig. 6. Regression with Fitts' Law (a) and with the modified $I D$ including a $\frac{T V}{D}$ term (b) 
We have seen in the previous section that movement time increases with TV but that this depends on distance: as distance increases, the degradation on movement time for a given value of TV decreases. Thus, the combined effect of TV and D may be captured by the ratio $T V / D$. We therefore consider the following model:

$$
M T=a+b \cdot I D+c \frac{T V}{D}
$$

The regression with this model yields $M T=171+174 . I D+237 . T V / D$ and a good adjusted $r^{2}=09414$. Taking advantage of the fact that $237 / 174 \approx 4 / 3$, we use the new index of difficulty $I D+4 / 3 . T V / D$ to plot the resulting data in Figure 6.b.

Of course, the validity of this model should be further tested and may be altered by considering larger sets of distances, target widths and DELAY conditions. Note that an important property of our experimental design is that the values for DELAY are shorter than the expected movement times in the Static condition. If TV is larger than the pointing time in the static condition, a different model is likely to apply since the user would have to move the pointer towards the memorized position of the target, wait until the target pops up (after $T V m s$ ), and finally acquire the target of width $W$ at a distance $\operatorname{err}(D)$ where $e r r$ is a function modeling the distance error when pointing to a memorized invisible target at a distance $D$. A candidate model for this situation could be $M T=T V+a+b \cdot \log _{2}(\operatorname{err}(D) / W+1)$, but this remains to be tested.

\section{Discussion and Future Directions}

The work presented in this paper is the first to study the acquisition of pop-up and animated targets. We did not find significant differences in performance between the acquisition of a static target and the acquisition of a "memorized" target that pops up immediately. However, we found a significant difference regarding errors in favour of static pointing and we also found that static pointing is significantly faster than pointing on animated and pop-up targets with a delay longer than $200 \mathrm{~ms}$. This suggests that delays and animations can indeed impair performance, and that techniques that keep the context (and the target) visible should be preferred.

We did not find significant performance differences between the acquisition of targets that pop up immediately and animated or pop-up targets with a delay of $200 \mathrm{~ms}$. However, we observed large differences when delays increase to 350 and $500 \mathrm{~ms}$, the magnitude of the difference being comparable to the increase in delay. This suggests that animations should be kept close to a duration of $200 \mathrm{~ms}$ whenever the acquisition of a target inside the animated object is desirable. Also, GUIs should do their best to keep the delay for popping up a potential target under $200 \mathrm{~ms}$. Note however that other design factors may come into play in the real world that are more important than pointing performance, such as the ability to perceive causality with animations.

Our study suggests that users are able to adapt their pointing movement dynamically. It is interesting to note that this adaptation depends on the feedback given to the user: animation affects the nature of the movement, slowing it down and leading to more sub-movements than pop-up. Since we did not observe a significant difference 
in performance between the acquisition of an animated target and a pop-up target (under the same delay) it is possible that users are able to take advantage of the target animation during the acquisition movement to better predict the final position of the target. We intend to pursue this hypothesis in future work.

Another area for future work is to extend the scope of the study. In this initial study, we conducted a "pure" experiment idealizing the real world. Now that we have a better understanding of the basic factors involved, we can start to examine more realistic situations, such as $2 \mathrm{D}$ pointing with real windows and icons. We also want to consider more complex tasks that involve pop-up/animated targets such as those described in section 3. Finally we need to study the effects of other factors such as the animation type, e.g., slow-in/slow-out, and the degree of position memorization. For pop-up targets, we also plan to investigate larger delays and the extreme case of memorized invisible targets in order to refine our movement time model.

\section{Acknowledgements}

We wish to thank the member of the InSitu lab and in particular Stéphane Huot, as well as our experiment participants and the anonymous reviewers for their feedback.

\section{References}

1. Accot, J., Zhai, S.: Refining Fitts' law models for bivariate pointing. In: Proc. Human Factors in Computing Systems (CHI 2003), pp. 193-200. ACM Press, New York (2003)

2. Aivar, M., Hayhoe, M., Chizk, C., Mruczek, R.: Spatial memory and saccadic targeting in a natural task. Journal of Vision 5(3:3), 177-193 (2005)

3. Appert, C., Beaudouin-Lafon, M.: SwingStates: Adding state machines to Java and the Swing toolkit. Software: Practice and Experience 38(11), 1149-1182 (2008)

4. Baudisch, P., Cutrell, E., Robbins, D., Czerwinski, M., Tandler, P., Bederson, B., Zierlinger, A.: Drag-and-pop and drag-and-pick: Techniques for accessing remote screen content on touch- and pen-operated systems. In: Proc. INTERACT 2003, pp. 57-64. IOS Press, IFIP (2003)

5. Bezerianos, A., Balakrishnan, R.: The vacuum: facilitating the manipulation of distant objects. In: Proc. Human Factors in Computing Systems (CHI 2005), pp. 361-370. ACM Press, New York (2005)

6. Bock, O., Eckmiller, R.: Goal-directed arm movements in absence of visual guidance: evidence for amplitude rather than position control. Exp. Brain Res. 62(3), 451-458 (1986)

7. Cao, X., Li, J.J., Balakrishnan, R.: Peephole pointing: Modeling acquisition of dynamically revealed targets. In: Proc. Human Factors in Computing Systems (CHI 2008), pp. 1699-1709. ACM Press, New York (2008)

8. Chapuis, O.: Gestion des fenêtres: enregistrement et visualisation de l'interaction. In: Proc. Journées Francophones d'Interaction Homme-Machine (IHM 2005), pp. 255-258. ACM, New York (2005)

9. Cockburn, A., Gutwin, C., Greenberg, S.: A predictive model of menu performance. In: Proc. Human Factors in Computing Systems (CHI 2007), pp. 627-636. ACM Press, New York (2007) 
10. Faure, G., Chapuis, O., Roussel, N.: Power tools for copying and moving: Useful stuff for your desktop. In: Proc. Human Factors in Computing Systems (CHI 2009), pp. 1675-1678. ACM Press, New York (2009)

11. Fitts, P.M.: The information capacity of the human motor system in controlling the amplitude of movement. Journal of Experimental Psychology 47, 381-391 (1954)

12. Flash, T., Henis, E.: Arm trajectory modifications during reaching towards visual targets. J. Cognitive Neuroscience 3(3), 220-230 (1991)

13. Gonzalez, C.: Does animation in user interfaces improve decision making? In: Proc. Human Factors in Computing Systems (CHI 1996), pp. 27-34. ACM Press, New York (1996)

14. Hinckley, K., Guimbretiere, F., Baudisch, P., Sarin, R., Agrawala, M., Cutrell, E.: The springboard: multiple modes in one spring-loaded control. In: Proc. Human Factors in Computing Systems (CHI 2006), pp. 181-190. ACM Press, New York (2006)

15. Hoffmann, E.R.: Capture of moving targets: a modification of Fitts' law. Ergonomics 34, 211-220 (1991)

16. Hornof, A.J., Kieras, D.E.: Cognitive modeling demonstrates how people use anticipated location knowledge of menu items. In: Proc. Human Factors in Computing Systems (CHI 1999), pp. 410-417. ACM Press, New York (1999)

17. Jeannerod, M.: The neural and behavioural organization of goal directed movements. Clarendon Press, Oxford (1988)

18. Kurtenbach, G., Buxton, W.: Issues in combining marking and direct manipulation techniques. In: Proc. User Interface Software and Technology (UIST 1991), pp. 137-144. ACM Press, New York (1991)

19. MacKenzie, I.S.: Fitts' law as a research and design tool in human-computer interaction. Human-Computer Interaction 7, 91-139 (1992)

20. Meyer, D., Smith, J., Kornblum, S., Abrams, R., Wright, C.: Optimality in human motor performance: Ideal control of rapid aimed movements. Psych. Review 95, 340-370 (1988)

21. Mould, D., Gutwin, C.: The effects of feedback on targeting with multiple moving targets. In: Proc. Graphics Interface (GI 2004), Canadian Hum.-Comp. Comm. Soc., pp. 25-32 (2004)

22. Obendorf, H., Weinreich, H., Herder, E., Mayer, M.: Web page revisitation revisited: Implications of a long-term click-stream study of browser usage. In: Proc. Human Factors in Computing Systems (CHI 2007), pp. 597-606. ACM, New York (2007)

23. Plamondon, R., Alimi, A.: Speed/accuracy trade-offs in target-directed movements. Behavioral and Brain Sciences 20(2), 279-349 (1997)

24. Schlienger, C., Conversy, S., Chatty, S., Anquetil, M., Mertz, C.: Improving users' comprehension of changes with animation and sound: An empirical assessment. In: Baranauskas, C., Palanque, P., Abascal, J., Barbosa, S.D.J. (eds.) INTERACT 2007. LNCS, vol. 4662, pp. 207-220. Springer, Heidelberg (2007)

25. Tabard, A., Mackay, W., Roussel, N., Letondal, C.: Pagelinker: integrating contextual bookmarks within a browser. In: Proc. Human Factors in Computing Systems (CHI 2007), pp. 337-346. ACM Press, New York (2007)

26. Thomas, B.H., Calder, P.: Animating direct manipulation interfaces. In: Proc. User Interface Software and Technology (UIST 1995), pp. 3-12. ACM Press, New York (1995)

27. Welford, A.T., Norris, A.H., Shock, N.W.: Speed and accuracy of movement and their changes with age. Acta. Psychologica 30, 3-15 (1969)

28. Zhai, S., Conversy, S., Beaudouin-Lafon, M., Guiard, Y.: Human on-line response to target expansion. In: Proc. Human Factors in Computing Systems (CHI 2003), pp. 177-184. ACM, New York (2003) 